\title{
Muon Bremsstrahlung and Muonic Pair Production in Air Showers.
}

\author{
A. N. Cillis ${ }^{\mathrm{a}}$ and S. J. Sciutto ${ }^{\mathrm{a} *}$ \\ ${ }^{a}$ Laboratorio de Física Teórica \\ Departamento de Física \\ Universidad Nacional de La Plata \\ C. C. 67 - 1900 La Plata
}

Argentina

The objective of this work is to report on the modifications in air shower development due to muon bremsstrahlung and muonic pair production. In order to do that we have implemented new muon bremsstrahlung and muonic pair production procedures in the AIRES air shower simulation system, and have used it to simulate ultra high energy showers in different conditions.

The influence of the mentioned processes in the global development of the air shower is important for primary particles of large zenith angles, while they do not introduce significant changes in the position of the shower maximum.

\section{Introduction}

The physics of ultra high energy cosmic rays plays an important role in our days. These primary particles can not be detected directly. When an ultra high energy astroparticle interacts with an atom of the Earth's atmosphere, it produces a number of secondary particles that continue interacting and generating more secondary particles. This process is generally known as the air shower.

We have developed a set of programs to simulate air showers and manage all the output data. Such simulating system is known as AIRES(AIRshower Extended Simulations) [1].

We started working on the topic of the electromagnetic processes in air showers some years ago. We analyzed the modification in the shower development due to the reduction of the electron bremsstrahlung and electron pair production by the LPM effect and the dielectric suppression [2]. We also studied the influence of the geomagnetic field in an air shower [3].

The main goal of this work is to analyze two processes that take place in an air shower: muon bremsstrahlung and muonic pair production (electron and positron). At energies high

\footnotetext{
${ }^{*}$ Fellow of CONICET (Argentina)
}

enough, these processes become more important than ionization, and therefore these mechanisms account for virtually all the energy losses for high energy muons.

In order to study the modifications that these effects introduce in an air shower, we developed new procedures for such mechanisms and incorporated them in the AIRES air shower simulation system [1]. The AIRES code has then been used as a realistic air shower simulator to generate the data used to make our analysis.

This work is organized as follows: we start in section 2 with a brief summary of the theory of the muon bremsstrahlung and muonic pair production; in section 3 we show the results of our simulations; finally we present our analysis and comments in the conclusion section.

\section{Theory}

\subsection{Theory of the Muon Bremsstrahlung}

The cross section for muon bremsstrahlung (MB) is calculated by the standard method of QED [4] similary as in the case of electron bremsstrahlung.

The first approach to the MB theory was due to Bethe and Heitler [5]. They considered in their calculation the screening of the atomic electrons. 
After this first formulation some corrections were introduced. Kelner, Kokoulin and Petrukhin [8] also included the bremsstrahlung with the atomic electrons. The nuclear form factor was investigated by Christy and Kusaka [9] for the first time and then by Erlykin [10]. Petrukhin and Shestakov 11] found that the influence of the nuclear form factor is more important than the ones predicted by the previous papers. This last results have been confirmed by Andreev et al. 12 who also considered the excitation of the nucleus.

\subsection{Theory of Muonic Pair Production}

In the lowest significant order of perturbation theory the muonic pair production(MPP) is a process of 4th order in QED.

Racah [13] was the first to calculate the MPP cross section in the relativistic region without taking into accuont the atomic and the nuclear form factor. Thereafter, Kelner [14 included the correction due to the screening of the atomic electrons. The analytical expression for any degree of screening was introduced by Kokoulin and Petrukhin 15]. Those authors also took into account 16] the correction due to the nuclear form factor. We wish to emphasize that the influence of the nuclear size is more important when the energy tranferred to the pair is large [16]. This last case is important for the air showers and therefore the nuclear size effect needs to be taken into account in the simulations.

\section{Air Showers Simulations}

We have calculated the total cross section, and equivalently, the mean free path (MFP), for both $\mathrm{MB}$ and MPP processes.

Figure 11 shows the MFP in $\mathrm{g} / \mathrm{cm}^{2}$ for MB, MPP and muon-nucleon interaction as functions of the kinetic energy of the initial muon. The information in this figure can be compared with the depth of the atmosphere. The vertical depth of the atmosphere is about $1000 \mathrm{~g} / \mathrm{cm}^{2}$ while in the case of very inclined showers ( $85^{\circ}$ of zenith angle) is about $9000 \mathrm{~g} / \mathrm{cm}^{2}$. Therefore, the probabilities of MB and MPP will not be very large except in the case of large zenith angles. The muonic

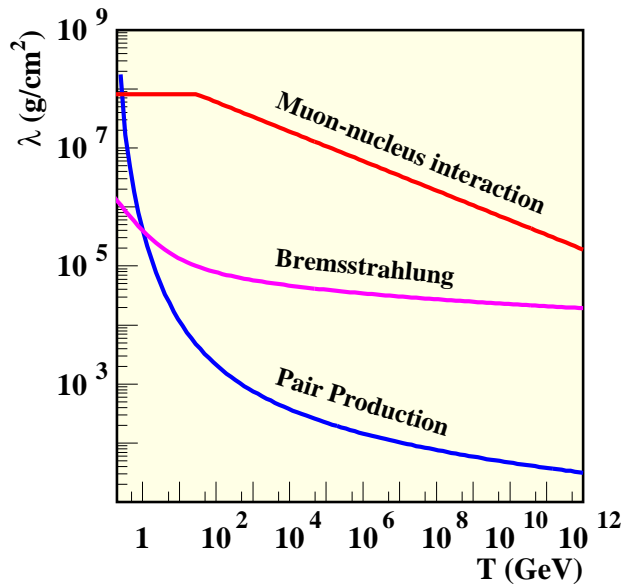

Figure 1. Mean free path: MB, MPP and muon nuclear interaction vs the kinetic energy of the initial muon.

component of the showers (number of muons divided by the number of electrons and gammas) at ground level becomes very important for zenith angles larger than $60^{\circ}$ (see, for example, figure 2 in reference [3]); this is another reason to expect that the influence of both effects will be more appreciable under those conditions.

The MFP's for MB and MPP diminish when the kinetic energy of the muon increases and therefore, the influence of both process will be more important for large energies.

Due to the fact that the MFP of muon-nucleus interaction 1 is larger (more than one order of magnitude) than the corresponding for $\mathrm{MB}$ we do not take this effect into account in our calculations.

In order to analyze the influence of $\mathrm{MB}$ and MPP in the development of air showers initiated by ultra high astroparticles we have performed simulations using the AIRES program [1] with different initial conditions: primary particles (protons, irons, muons), primary energies $\left(10^{18}\right.$, $10^{19}$ and $3 \times 10^{20} \mathrm{eV}$ ) and zenith angles.

\footnotetext{
${ }^{2}$ We used the same parametrization of the total cross section used by GEANT 17
} 
To start with the analysis of the simulated data, let us consider the case of a single muon (eventually produced during the development of a given shower). This particle may generate a secondary shower if the processes of MB and MPP are taken into account. This effect is clearly illustrated in figure 2a where the longitudinal development of all charged particles is plotted versus the slant depth. In this case the initial particle is a muon of $10^{14} \mathrm{eV}$. When MB and MPP are not taken into account there is practically no production of new particles. On the other hand, when the effects are considered, it is possible to appreciate a secondary shower.

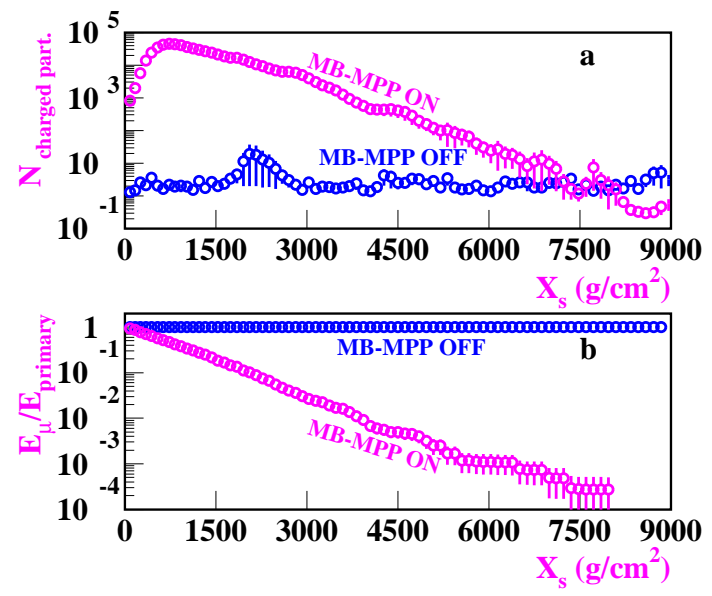

Figure 2. a)Longitudinal development of all charged particles vs the slant depth. Primary particle (Energy): muon $\left(10^{14} \mathrm{eV}\right)$. b)Longitudinal development of muon fractional energy vs the slant depth.

In figure $2 \mathrm{~b}$ the longitudinal development of the muon fractional energy (muon energy/primary energy) is plotted. When the effects are not taken into account the muon practically does not loss energy during all its path, while if the effects are considered, the muon energy loss is significant.

We have also studied the modifications in the global observables of the shower, for example when the primary particle is a proton.

We have plotted in figures 3 and 14 different obsevables for $3 \times 10^{20} \mathrm{eV}$ proton shower with zenith angle $85^{\circ}$, taking and not taking into a account the effects of MB and MPP. Figure 3 represents the longitudinal development of electrons and positrons $\left(e^{+/-}\right)$versus the slant depth. The number of $e^{+/-}$increase when the effects are taken into account. The differences between the two cases are clearly noticeable in the tail of the showers. The longitudinal development in energy

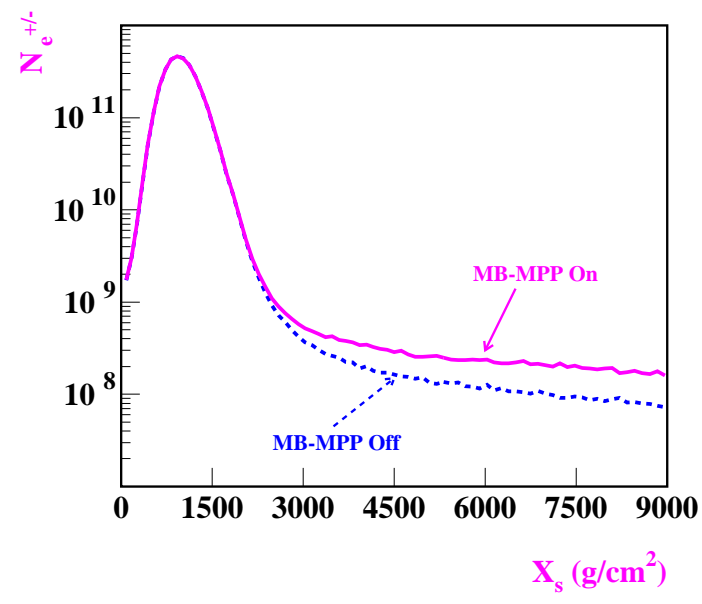

Figure 3. Longitudinal development of electrons and positrons vs the slant dept. Primary particle (Energy) proton $\left(3 \times 10^{20} \mathrm{eV}\right)$.

of muons is plotted in figure 1 . In this case the difference is about $15 \%$ at the tail. The energy of muons diminishes when the effects are considered.

For the longitudinal development of muons versus the slant depth the difference between the cases taking and not taking into account the effects is less significant, about $2 \%$ (not plotted here).

The modification in the shower development due to the MB and MPP diminishes for small zenith angle of the primary particle. We did not find significant modification of the shower development for zenith angles smaller than $45^{\circ}$. 


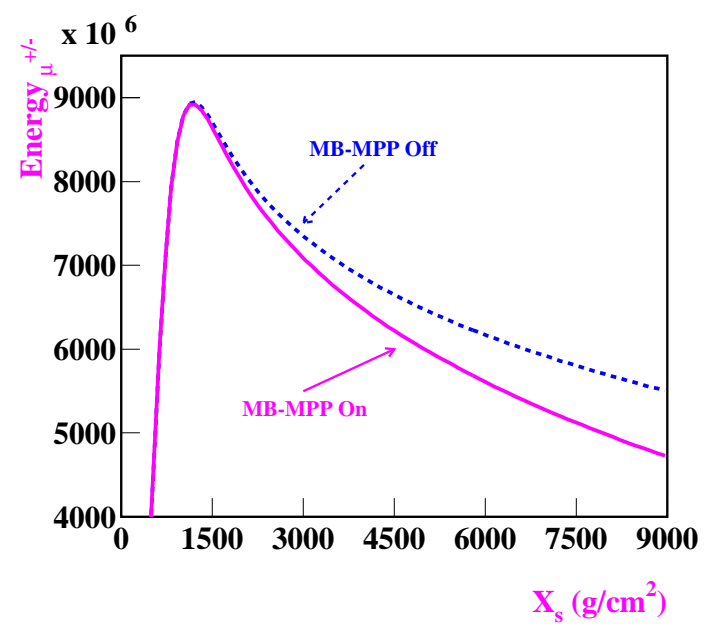

Figure 4. Longitudinal development in energy of muon vs the slant depth. Primary particle (Energy) proton $\left(3 \times 10^{20} \mathrm{eV}\right)$.

\section{Conclusions}

Due to MB and MPP, a high energy muon in the shower can generate a secondary shower. This practically does not occur if the mentioned mechanisms are not taken into account.

The influence of MB and MPP in the total development of the air showers is more important for primary particle of large zenith angles. Below zenith angles of 45 degrees we do not observe any significant difference between the cases where these effects are or not taken into account.

The MB and MPP do not generate visible modifications in the position of the maximum development of the shower. The modification that we observe affect the shower development well past its maximum (tail of the shower).

We are performing more simulations in order to make a complete study of the influence of $\mathrm{MB}$ and MPP in an air shower [18].

\section{Acknowledgments}

This work was partially supported by CONICET, Agencia Nacional de Programación Científica and FOMEC program, Argentina.

\section{REFERENCES}

1. S. J. Sciutto, AIRES, a system for air shower simulations. User's manual and reference guide, version 2.2.0, preprint astroph 9911331 (1999). The AIRES software and documentation are also available electronically at the following Web address: www.fisica.unlp.edu.ar/auger/aires.

2. A. N. Cillis, H. Fanchiotti, C. A. Garcia Canal and S. J. Sciutto, Phys. Rev. D 59 (1999) 113012.

3. A. N. Cillis and S. J. Sciutto, Journal of Physics G: Nuclear and Particle Physics 26 Number 3 (2000) 309.

4. W. Greiner and J. Reinhardt, Quantum Electrodynamics, Springer-Verlag (1992).

5. H. A. Bethe, Proc. Cambridge Phil. Soc. 30 (1934) 524.

6. H. A. Bethe and W. Heitler, Proc. Roy. Soc. (London) 146 (1934) 83.

7. B. Rossi, High Energy Particles, Prentice Hall (1956).

8. S. R. Kelner, R. P. Kokoulin and A. A. Petrukhin, Phys. Atomic Nuclei 60 (1997) 576.

9. R. F. Christy and S. Kusaka, Phys. Rev. 59 (1941) 405.

10. A. D. Erlykin, Izv. Akad. Nauk SSSR, Ser. Fiz. (1965) 291757.

11. A. A. Petrukhin and V. V. Shestakov, Canadian Journal of Physics 46 (1967) s377.

12. Yu M. Andreyev, L. B. Bezrukov E. V. and Bugaev, Phys. Atomic Nuclei 57 (1994) 2066.

13. G. Racah, Nuovo Cimento 14 (1937) 93.

14. S. R. Kelner Yadernaya Fiz., 5 (1967) 1092.

15. R. P. Kokoulin and A. A. Petrukhin, Proc. 11th. Intern. Conf. on Cosmic Rays, Budapest, Acta Phys. Acad. Sci. Hung., 29 Suppl. 4 (1969) 277.

16. R. P. Kokoulin and A. A. Petrukhin, Proc. 12th. Intern. Conf. on Cosmic Rays, Hobart 6 (1971) 2436.

17. GEANT: CERN Program library Long Writeup W5013 (1994).

18. A. N. Cillis and S. J. Sciutto, in progress. 